\title{
TRAQUELECTOMÍA LAPAROSCÓPICA DESPUÉS DE HISTERECTOMÍA SUBTOTAL: REPORTE DE DOS CASOS EN MEDELLÍN, COLOMBIA
}

\section{A report of two cases of using laparoscopic trachelectomy following subtotal hysterectomy in Medellin, Collombia}

José F. De Los Ríos, M.D. *, Juan C. Ochoa, M.D.**, Juan D. Castañeda ***

Recibido: julio 17/07 - Aceptado: junio 6/08

\section{RESUMEN}

Objetivo: reportar dos casos de traquelectomía por laparoscopia.

Institución: Unidad de Cirugía Endoscópica Ginecológica, Clínica del Prado, Medellín, Colombia.

Diseño: se presenta el reporte de dos pacientes con muñón cervical sintomático después de histerectomía subtotal y su manejo por vía laparoscópica.

Variables: se describen los datos demográficos de las pacientes, el diagnóstico prequirúrgico, la técnica empleada para la remoción del cérvix y los datos de la evolución posoperatoria.

Conclusiones: la evidencia disponible cataloga el abordaje laparoscópico como seguro para la realización de traquelectomía en casos de enfermedad benigna. Este es el primer reporte de traquelectomía laparoscópica publicado en la literatura colombiana.

Palabras clave: muñón cervical, histerectomía subtotal, laparoscopia, traquelectomía.

* Ginecólogo Endoscopista, Unidad de Cirugía Endoscópica Clínica del Prado. Profesor de Endoscopia Ginecológica CES, Medellín, Colombia. Correo elctrónico: cristidelos@une.net.co

** Profesor de Endoscopia Ginecológica CES, Medellín, Colombia.

*** Fellow de Endoscopia Ginecológica CES, Medellín, Colombia.

\section{SUMMARY}

Objective: reporting 2 cases of laparoscopic trachelectomy.

Setting: the Gynaecological Endoscopy Unit at the Clínica del Prado in Medellín, Colombia.

Design: this is a case report concerning two patients having symptomatic cervical stump following subtotal hysterectomy and its management by laparoscopy.

Outcomes: the patients' demographic data, pre-operation diagnosis, the surgical technique used for removing the cervix and the postoperative results are described.

Conclusions: the available data revealed that a laparoscopic approach is a safe alternative for trachelectomy in benign conditions. This is the first report of laparoscopic trachelectomy published in Colombian literature.

Key words: cervical stump, subtotal hysterectomy, laparoscopy, trachelectomy.

\section{INTRODUCCIÓN}

La práctica de la histerectomía subtotal en Colombia está limitada a cirugías complejas, donde por las condiciones anatómicas o patológicas de la paciente no es posible exponer adecuadamente el cérvix para su resección o para algunos casos de 
histerectomía obstétrica urgente, ya que se prefiere evitar la extirpación cervical para no aumentar el sangrado, el tiempo quirúrgico y la morbilidad de una paciente que usualmente se encuentra en estado crítico.

El hecho de que nuestro país sea considerado como zona endémica, ${ }^{1}$ para la presentación de cáncer cervical, aleja más la posibilidad de su instauración como manejo preferencial en casos de enfermedad uterina benigna. Dada la infrecuente realización de histerectomías subtotales en nuestro medio, no conocemos datos contundentes sobre el comportamiento de los muñones cervicales residuales, pero en otras regiones el tema es común.

Se ha descrito que hasta el 24,3\% de las mujeres, después de la histerectomía subtotal, presentan molestias atribuibles a la presencia del cérvix tales como: dispareunia, dolor pélvico crónico, sangrado cíclico y leucorrea sintomática, problemas que en muchos casos requieren procedimientos quirúrgicos adicionales para el manejo de los síntomas. ${ }^{2,3,4}$ También han sido informadas complicaciones graves como la necrosis del muñón cervical residual con choque séptico concomitante. ${ }^{5}$

Cuando ocurren problemas como estos, una de las opciones terapéuticas es la traquelectomía y esta puede realizarse por vía vaginal, por vía abdominal o por vía laparoscópica, dependiendo de las características de la paciente y del entrenamiento del cirujano. Se encuentran pocos reportes acerca de la realización de las traquelectomías por enfermedad benigna que utilizan esta última vía. El propósito de este trabajo es reportar los dos primeros casos de traquelectomía laparoscópica por patología benigna del muñón publicados en la literatura colombiana y discutir las ventajas que este abordaje puede brindar en casos semejantes.

\section{REPORTE DE CASOS}

\section{Caso 1}

Paciente de 35 años de edad, remitida a la unidad de endoscopia ginecológica de la Clínica del Prado, institución privada, ubicada en Medellín, Colom- bia, en junio del 2006 por dolor pélvico acíclico y dispareunia profunda severa de varios años de evolución. Antecedente de histerectomía subtotal de emergencia ocho años antes, por presentar una hemorragia obstétrica masiva. El examen físico no reveló prolapso ni masas pélvicas, pero al tacto vaginal se evidenció un exquisito dolor a la movilización del muñón cervical remanente que según la paciente, era semejante a la molestia que sentía durante el coito. Ante estos hallazgos, se decidió programar entonces una traquelectomía laparoscópica.

Después de aplicar un colpotomizador V-care (ConMed corporation) en el muñón cervical por vía vaginal, se realizó una inspección laparoscópica encontrándose el muñón cervical cubierto por peritoneo y fijo a los ligamentos uterosacros, sin evidencia de endometriosis, ni adherencias.

Se incidió transversalmente el peritoneo sobre el muñón cervical, disecando la vejiga y el recto, hasta evidenciar por completo la copa cervical del colpotomizador, la cual fue constantemente presionada por el ayudante en dirección craneal, para facilitar la disección y alejar los uréteres del campo quirúrgico. Se procedió a hacer electrocoagulación y corte de los vasos uterinos con 25 vatios de corriente bipolar y tijeras, y luego se realizó una colpotomía circular con 60 vatios de corriente monopolar sobre el borde externo de la copa cervical, logrando la extirpación completa del muñón residual y extrayéndolo por vía vaginal sin dificultad. Se reconstruyó el anillo pericervical y se reafrontó la mucosa vaginal en dos planos continuos intracorpóreos con poliglactina 2 ceros, sin ninguna complicación. El sangrado total estimado fue menor de $15 \mathrm{cc}$. El tiempo de la cirugía fue de 50 minutos. El estudio de patología reportó cervicitis crónica. La revisión posoperatoria fue completamente normal. Diez meses después de realizada la cirugía, la paciente relata sentirse satisfecha ya que su dolor acíclico disminuyó notoriamente, su dispareunia desapareció y al examen físico se encuentra una cúpula alta y fija. 


\section{Caso 2}

Paciente de 44 años de edad, quien consultó por presentar sensación de masa que protruía por la vagina, sin sintomatología que evidenciara disfunción urinaria, defecatoria o sexual. Era hipertensa y dos años antes le habían practicado una histerectomía abdominal reportada como muy difícil por la obesidad y una cistouretropexia tipo Burch. La paciente desconocía que el procedimiento había sido subtotal. $\mathrm{Al}$ examen físico pesaba 90 kilos y tenía un índice de masa corporal de 32. El examen vaginal evidenciaba un prolapso del cérvix uterino que alcanzaba el himen sin sobrepasarlo, sin compromiso de los compartimientos anterior ni posterior. El personal de la Unidad de Cirugía Endoscópica de la Clínica del Prado, concluyó que por las dificultades reportadas en la histerectomía, la paciente tenía factores de riesgo para la formación de adherencias y que para poder practicar una adecuada reparación del sistema de soporte apical en una paciente obesa era mejor una visualización directa de las estructuras. Por esta razón, se decidió llevar a cabo la intervención por vía laparoscópica. Se aplicó un colpotomizador V-care (ConMed corporation) en el muñón cervical por vía vaginal y se realizó una inspección laparoscópica encontrando una pelvis totalmente bloqueada por adherencias. Se efectuó adhesiolisis con bipolar y tijera hasta exponer adecuadamente los órganos pélvicos. Se incidió y se disecó el peritoneo sobre el muñón cervical y se reconocieron los fórnices vaginales gracias a la presión ejercida por la copa cervical del colpotomizador. Se localizaron ambos ligamentos uterosacros y se marcaron con seda 2 ceros. Con 30 vatios de corriente bipolar se coagularon y seccionaron los vasos uterinos que aún cabalgaban sobre los aspectos laterales del muñón. Con corriente monopolar se realizó colpotomía circular y se extrajo el cérvix. Se suturó la mucosa vaginal con un plano continuo intracorpóreo con poliglactina cero y posteriormente se procedió a practicar la reparación del anillo pericervical con una sutura continua intracorpórea de poliéster 0 que incluyó la fascia pubocervical, la fascia rectovaginal y el complejo uterosacro-cardinal a cada lado. Finalmente se efectuó una plicatura de los ligamentos uterosacros previamente marcados, con poliéster 0 , corrigiendo el defecto de soporte apical. La evolución posoperatoria fue satisfactoria y la paciente fue dada de alta cuatro horas después del procedimiento. Anatomía patológica reportó una cervicitis crónica. Tres meses después de la resección, la paciente se encuentra asintomática, satisfecha y sin prolapso residual. Al examen físico se evidencia una cúpula alta y adecuadamente suspendida. No se presentaron complicaciones posoperatorias.

\section{DISCUSIÓN}

La remoción del muñón cervical es una cirugía poco común para el ginecólogo. Su indicación más frecuente es el prolapso (52\%), hecho que cuestiona la teoría de que dejar el cérvix in situ evita futuros prolapsos. ${ }^{2}$ La publicación que reporta el mayor número de casos presenta el análisis de 310 cirugías en 30 años. $^{2}$ En este estudio, el 65\% de las traquelectomías se llevaron a cabo por la vía vaginal y el resto por laparotomía. Cuatro casos fueron realizados por laparoscopia, pero no fueron incluidos en el análisis porque el trabajo trataba de evaluar solo las diferencias entre las vías vaginal y abdominal. Hay pocos reportes de la realización de traquelectomías por enfermedad benigna utilizando laparoscopia. Los primeros seis casos fueron descritos por Nezhat ${ }^{6}$ en 1996, luego aparecieron 14 referenciados por Okaro. ${ }^{3}$ El 53\% de mujeres con muñón cervical llevadas a cirugía por dolor o dispareunia tenían hallazgos patológicos adicionales como endometriosis del cérvix o adherencias del mismo, entre otros, que no habrían sido diagnosticados ni tratados en caso de haber sido operadas por la vía vaginal. Incluso hay reportes recientes de siete traquelectomías radicales por laparoscopia en pacientes con carcinomas del muñón cervical. ${ }^{7,8}$ No se ha reportado ninguna complicación de la traquelectomía laparoscópica, lo que sugiere que se trata de un procedimiento seguro.

Dado que el prolapso del muñón cervical puede clasificarse como un defecto del compartimiento api- 
cal y por lo tanto, corresponde a una falla en el nivel I de DeLancey, debe llevarse a cabo para su adecuado manejo una reparación apropiada de los componentes del anillo pericervical, lo cual no siempre es fácil de realizar por la vía vaginal. En el caso concreto de nuestra segunda paciente, el objetivo de reparar correctamente esta estructura, fue lo que nos motivó a optar por la vía laparoscópica. En nuestra opinión, la vía endoscópica es una alternativa para considerar ya que al parecer es segura para la extracción del muñón cervical retenido y sintomático. Ofrece ventajas sobre la vía vaginal como lo son: la visualización directa y magnificada de la anatomía pélvica para reconstruir adecuadamente las estructuras de sostén apical y la probabilidad de diagnosticar y tratar patologías asociadas como las adherencias o la endometriosis del muñón. Además evita la morbilidad de la laparotomía y permite a la paciente disfrutar de las conocidas ventajas de la cirugía de accesos mínimos como lo son: procedimiento ambulatorio, menor dolor, más rápida recuperación, menor necesidad de analgésicos y menor área de cicatriz.

\section{REFERENCIAS}

1. García-Soto G, Pachón-Rincón JJ, Meneses-Cubides RS, Zuleta-Tobón JJ. Cáncer de cuello uterino: experiencia durante un periodo de seis años en un hospital universitario colombiano. Rev Colomb Obstet Ginecol 2007;58:21-7.

2. Hilger WS, Pizarro AR, Magrina JF. Renoval or the retained cervical stump. Am J Obstet Gynecol 2005;193:2117-21.

3. Okaro EO, Jones KD, Sutton C. Long term outcome following laparoscopic supracervical hysterectomy. BJOG 2001;108:1017-20.

4. van der Stege JG, van Beek JJ. Problems related to the cervical stump at follow-up in laparoscopic supracervical hysterectomy. JSLS 1999;3:5-7.

5. Huang JY, Ziegler C, Tulandi T. Cervical stump necrosis and septic shock af ter laparoscopic supracervical hysterectomy. J Minim Invasive Gynecol 2005;12: 162-4.

6. Nezhat CH, Nezhat F, Roemisch M, Seidman DS, Nezhat C. Laparoscopic trachelectomy for persistent pelvic pain and endometriosis after supracervical hysterectomy. Fertil Steril 1996;66:925-8.

7. Liang Z, Xu H, Chen Y, Li Y, Chang Q, Shi C. Laparoscopic radical trachelectomy or parametrectomy and pelvic and para-aortic lymphadenectomy for cervical or vaginal stump carcinoma: report of six cases. Int J Gynecol Cancer 2006;16:1713-6.

8. Diaz-Feijoo B, Gil-Moreno A, Puig O, MartinezPalones JM, Xercavins J. Total laparoscopic radical trachelectomy with intraoperative sentinel node identification for early cervical stump cancer. J Minim Invasive Gynecol 2005;12:522-4. 